\title{
Smooth Decomposition of Generalized Fatou Set Explains Smooth Structure in Generalized Mandelbrot Set
}

\author{
J. Peinke, J. Parisi, and B. Röhricht \\ Physical Institute (Experimental Physics II), University of Tübingen \\ O. E. Rössler \\ Institute for Physical and Theoretical Chemistry, University of Tübingen \\ W. Metzler \\ Interdisciplinary Working Group Mathematization, University of Kassel \\ Z. Naturforsch. 43 a, 14-16 (1988); received October 15, 1987
}

Generalized Mandelbrot sets arise in perturbed (non-analytic) versions of the complex logistic map. Numerically, it contains smooth portions as shown previously. To exclude that this result is specific to particular initial conditions only, the structure of the analogue to the Fatou set is looked at in the region in question. The set of non-divergent points is being "eaten up" by a smooth invading boundary. Therefore, the same type of decomposition applies independent of position in parameter space, in the region in question.

\section{Introduction}

The Mandelbrot set is a boundary in parameter space. It arises in the complex logistic map. It separates those points for which there exists an attractor in the finite from the rest. A single point in the space of initial conditions, the origin, suffices to be investigated in the analytic case as is well known (see [1] for a review). Recently, we showed that a perturbation away from analyticity leads to a modified, "generalized" Mandelbrot set [2]. Unfortunately, it then no longer suffices to investigate a single initial condition over the whole parameter space. Therefore, we could not exclude that our main result found, the presence of smooth portions in the set, was an artifact in the sense that it would occur only for specific initial conditions.

The decisive question is the behavior of the basin boundary. This boundary in the analytic case contains the Fatou set. There is a connection, not yet completely understood, between the fractal structure of the Fatou sets on the one hand and the local structure of the Mandelbrot set on the other [3]. If the Fatou set acquires smooth portions away from analyticity, this might explain a corresponding property of the generalized Mandelbrot set.

Reprint requests to Jürgen Parisi, Physikalisches Institut, Lehrstuhl Experimentalphysik II, Universität Tübingen, Morgenstelle 14, D-7400 Tübingen, F.R.G.

\section{Results}

A smooth region of the generalized Mandelbrot set was investigated. It is indicated in Figure 1. We scanned across the boundary along several parallel lines.

Moving across the smooth boundary, we obtained the pictures of Figure 2. At first the "Fatou set" is singly connected (Figure 2a). Then it decomposes successively into more and more strongly disconnected pieces, in a $2^{n}$-type bifurcation. Unexpectedly, the corresponding scaling constant is close to unity (over 7 bifurcations tested in sequence). Two features of this decomposition are unexpected. On the one hand, the disconnectedness, on the other the fact that
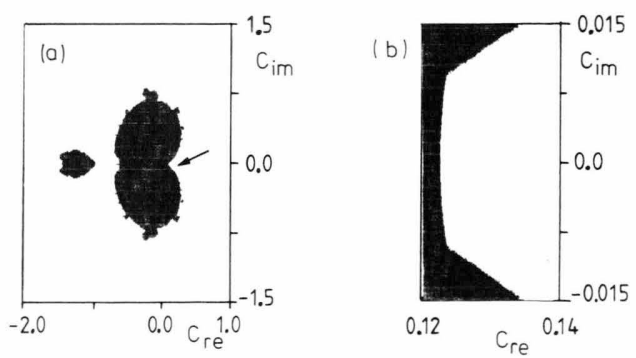

Fig. 1. (a) The generalized Mandelbrot set. Non-divergent points in the parameter space of the perturbed version of the complex logistic map, $x_{n+1}=x_{n}^{2}-y_{n}^{2}+a x_{n}+c_{\text {re }}, y_{n+1}=$ $2 x_{n} y_{n}+c_{\text {im }}$ for $a=0.3$, are plotted black (initial condition $x_{0}=y_{0}=0 ; 500$ iteration steps). (b) Blow-up of the parameter region shown in (a). 

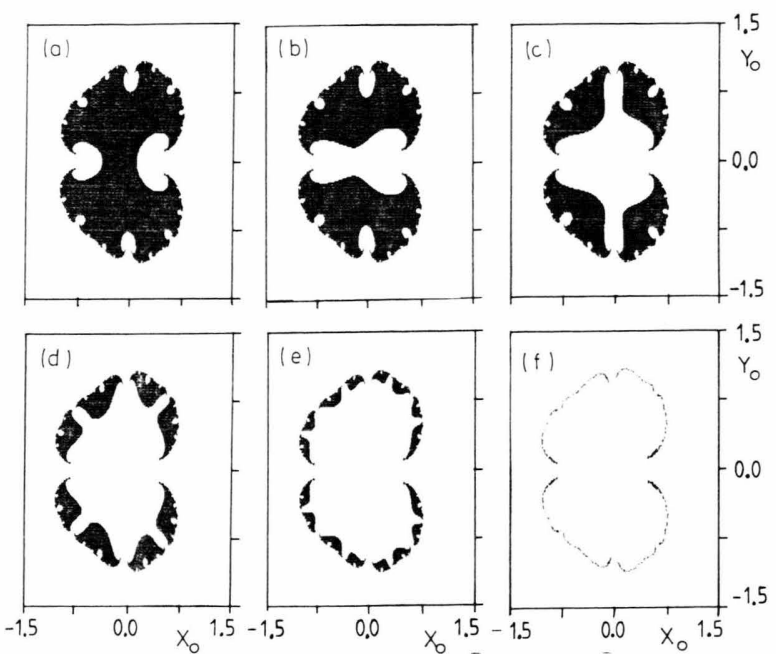

Fig. 2. Sequence of generalized Fatou sets. According to the generalized Mandelbrot set in Fig. 1, non-divergent points in the space of initial conditions are plotted black. The parameter values are chosen as follows: $c_{\mathrm{re}}=0.12253905$ (a), $c_{\mathrm{re}}=0.12253925$ (b), $c_{\mathrm{re}}=0.12253940$ (c), $c_{\mathrm{re}}=0.12253955$ (d), $c_{\text {re }}=0.12253975(\mathrm{e})$, and $c_{\mathrm{re}}=0.12254020(\mathrm{f}) ; c_{\mathrm{im}}=0$ is always kept constant.

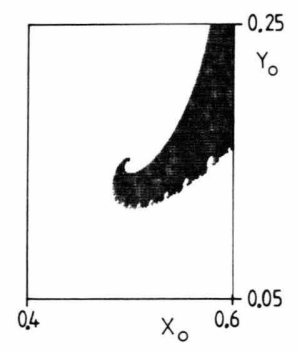

Fig. 3. Blow-up of a region of the generalized Fatou set shown in Figure 2e.
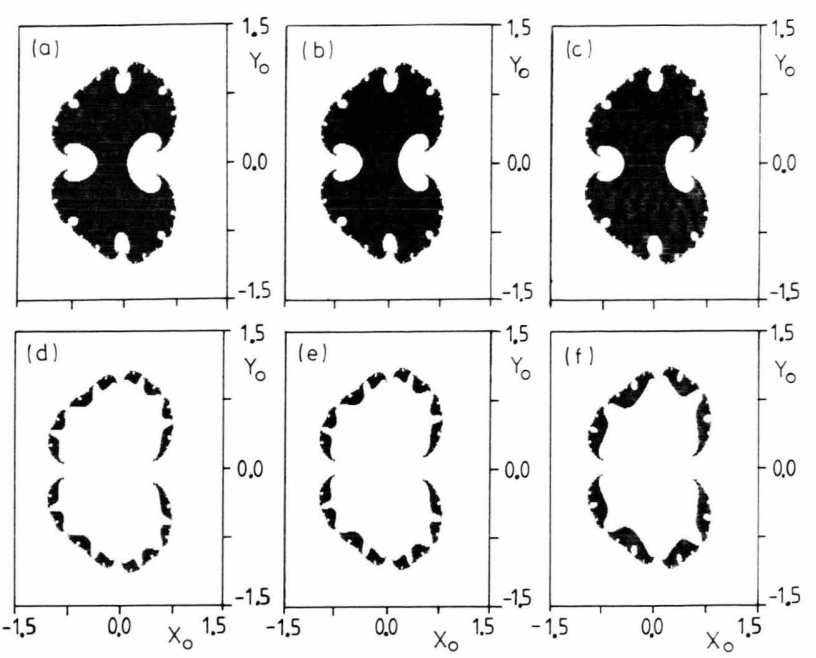

Fig. 4. Dependence of generalized Fatou sets upon the orthogonal parameter $c_{\mathrm{im}}$ for two different values of $c_{\mathrm{re}}$ : $c_{\mathrm{im}}=0 \quad$ (a), $\quad c_{\mathrm{im}}=0.00005 \quad$ (b), $c_{\mathrm{im}}=0.00010 \quad$ (c); $\quad c_{\mathrm{re}}=$ 0.12253905 is kept constant $(\mathrm{a}-\mathrm{c}) ; c_{\mathrm{im}}=0(\mathrm{~d}), c_{\mathrm{im}}=0.00005$ (e), $c_{\mathrm{im}}=0.00010(\mathrm{f}) ; c_{\mathrm{re}}=0.12253975$ is kept constant $(\mathrm{d}-\mathrm{f})$. all boundaries coming to lie inside the confines of the previously connected set are smooth (see Fig. 3 for a blow-up of a detail from Figure 2e).

Furthermore, it turns out that the outer boundary of the sets of Fig. 2 are virtually unchanged (to better than 1 percent) during the whole bifurcation sequence. That is, only the "invading" smooth internal boundary is responsible for the decay of the Fatou set.

This result is rather insensitive, on the other hand, $\left(c_{\mathrm{im}}\right)$, not across but rather parallel to the boundary. This is documented in Figure 4. to major variations in the second local parameter

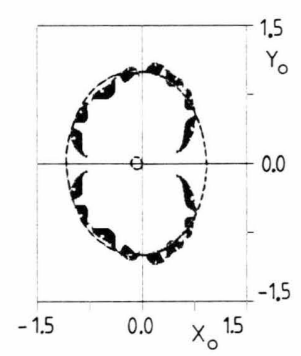

Fig. 5. Illustration of the critical circles relative to an exemplary Fatou set $\left(c_{\mathrm{re}}=0.12253975, c_{\mathrm{im}}=0\right)$. The inner circle, $(x+a / 4)^{2}+y^{2}=(a / 4)^{2}$, corresponds to the Jacobian determinant equal to zero, while the outer circle, $(x+a / 4)^{2}+y^{2}=1$ $+(a / 4)^{2}$, corresponds to the Jacobian determinant equal to one.
A little analytical result is finally presented in Figure 5. There the contraction of the map (Jacobian determinant smaller than or equal to one) is plotted - a circle -, with the corresponding Fatou set shown in the same picture. One sees that the latter can with parts lie outside. This reflects the fact that the corresponding attractor is periodic so that a net contraction is nevertheless possible. The Figure also shows that the points of infinite contraction (Jacobian determinant equal to zero) need not lie within the Fatou set. 


\section{Discussion}

A new result found numerically was presented. A "Fatou analogous" set can decompose in a perioddoubling bifurcation sequence into disconnected fragments. This sequence occurs very rapidly, over a very short range of parametric variation, with a very unusual scaling constant only slightly larger than unity. This decomposition, moreover, involves only smooth, nonfractal curve segments. It also is "locally universal" in the sense of remaining virtually unchanged over a "large" variation of the orthogonal parameter. This result at the same time can explain, in qualitative terms, the existence of smooth portions of the generalized Mandelbrot set. We felt the result may elucidate

1] P. Blanchard, Bull. Amer. Math. Soc. 11, 85 (1984).

[2] J. Peinke, J. Parisi, B. Röhricht, and O. E. Rössler, Z. Naturforsch. 42a, 263 (1987). the mutual relationship between fractal structures in parameter and in phase space.

It appears that the non-analytic fractal phenomena are also a rich field of investigation. There is hope that due to the greater simplicity of some of the phenomena occurring here, a deeper understanding of the analytic case - which is embedded in the present neighborhood like a slice through a tree trunk [2] - may also be possible eventually.

\section{Acknowledgements}

We thank Rudolf P. Huebener and Claus Kahlert for discussions. J. Pe. and B. R. thank the VW Foundation for the support of this work.

[3] H. O. Peitgen and P. H. Richter, The Beauty of Fractals, Springer-Verlag, Berlin 1986. 